\title{
ADF and cofilin-1 collaborate to promote cortical actin flow and the leader bleb-based migration of confined cells
}

Maria F. Ullo and Jeremy S. Logue*

Department of Regenerative and Cancer Cell Biology, Albany Medical College, 47 New

Scotland Ave, Albany, NY 12208, USA

*Corresponding author

$\underline{\text { loguej@mail.amc.edu }}$

Running head: ADF and cofilin-1 collaborate to promote cortical actin flow 


\begin{abstract}
Melanoma cells have been shown to undergo fast amoeboid (leader bleb-based) migration, requiring a single large bleb for migration. In leader blebs, is a rapid flow of cortical actin that drives the cell forward. Using RNAi, we find that co-depleting cofilin-1 and ADF led to a large increase in cortical actin, suggesting that both proteins regulate cortical actin. Furthermore, severing factors can promote contractility through the regulation of actin architecture. However, RNAi of cofilin-1 but not ADF led to a significant decrease in cell stiffness. We found cofilin-1 to be enriched at leader bleb necks, whereas RNAi of cofilin-1 and ADF reduced bleb sizes and the frequency of motile cells. Strikingly, cells without cofilin-1 and ADF had blebs with abnormally long necks. Many of these blebs failed to retract and displayed slow actin turnover. Collectively, our data identifies cofilin-1 and ADF as actin remodeling factors required for fast amoeboid migration.
\end{abstract}

Keywords: cancer, metastasis, cell migration, amoeboid, cytoskeleton, actin, ADF, cofilin-1 


\section{Introduction}

Cell migration requires tight spatiotemporal control of the Filamentous-actin (F-actin) cytoskeleton. For mesenchymal migration, actin assembly/disassembly and myosin contraction must occur within specific regions of the cell (1). Relative to other, recently described modes of migration, the mechanisms by which mesenchymal cells coordinate these processes are reasonably well understood. Whereas, in amoeboid cells, which migrate using intracellular driven protrusions of the Plasma Membrane (PM) or blebs, the mechanisms conferring spatiotemporal control of the F-actin cytoskeleton are not well known.

Within tissues, cells encounter a variety of physicochemical environments. We and others discovered that in response to tissue confinement, cancer cells frequently undergo a phenotypic transition to what was termed, fast amoeboid migration (2-5). A hallmark of amoeboid migration is the presence of blebs, which form as a result of PM-cortical actin separation (6). Typically, blebs are rapidly retracted following the reassembly of cortical actin on bleb membranes and the recruitment of myosin (6). However, during fast amoeboid migration, cells form a very large and stable bleb. Within these blebs, is a cortical actin network that flows from the bleb tip to the neck, which separates leader blebs from the cell body (2-5). Together with non-specific friction, flowing cortical actin provides the motive force for cell movement (4). Accordingly, we simultaneously termed this mode of migration, Leader BlebBased Migration (LBBM) (2). Because migration plasticity is thought to be a major contributor of metastasis, our aim here is to identify the essential factors required for the rapid cortical actin flow in leader blebs.

Previous studies have demonstrated that myosin is enriched at the neck of leader blebs. Indeed, the myosin at this location has been shown to be required for rapid cortical actin flow (35). However, what drives the disassembly of F-actin at the neck to replenish the pool of G-actin at the leader bleb tip is unknown. Thus, we hypothesize that actin disassembly factors within leader blebs play a pivotal role in this process. Similarly, in mesenchymal cells, retrograde actin flow is driven by both myosin contraction and actin disassembly, the latter of which is accelerated by the ADF/cofilin family of severing factors (7). Severing depends on ADF/cofilin inducing a structural change within the actin filament, an activity known to be repressed by LIM Kinase (LIMK) phosphorylation on Ser 3 (8). However, the consequence of actin severing is variable, depending on the availability of barbed end capping proteins. At a sufficiently high level, new barbed ends are capped, promoting actin disassembly from new pointed ends (9). Conversely, if left uncapped, increased polymerization from new barbed ends may occur (9). Additionally, by competing for F-actin binding (e.g., Arp2/3), ADF/cofilin can regulate actin architecture (10). The effects of ADF/cofilin on cortical actin are even less certain, as the focus of many studies has been on the actin within lamellipodia. Therefore, we set out to determine the role of $\mathrm{ADF} /$ cofilin in regulating the cortical actin cytoskeleton in fast amoeboid cells. 
By combining an in vitro assay for the precise confinement of cells with quantitative imaging approaches, we report that ADF and cofilin-1, together, are required for the rapid disassembly of incoming cortical actin at leader bleb necks. Under conditions of confinement, we find that melanoma and lung adenocarcinoma cells depleted of both proteins display dramatic defects in bleb morphology and dynamics. Consequently, cells without ADF and cofilin-1 cannot undergo LBBM. Thus, we reveal unanticipated roles for ADF and cofilin-1 in driving confined (leader bleb-based) migration.

\section{Results}

\section{ADF and cofilin-1 are required for leader bleb-based migration}

Using our previously described approach for cell confinement, which involves placing cells under a slab of PDMS held at a defined height $(\sim 3 \mu \mathrm{m})$ above cover glass, cancer cells will switch to LBBM (Fig. 1A \& Movie S1) (11). Moreover, within leader blebs, we find a rapid flow of cortical actin, which together with non-specific friction, provides the motive force for cell movement (Fig. 1B \& Movie S2) (5). As indicated by an enrichment in EGFP tagged Regulatory Light Chain (EGFP-RLC), we observe a concentration of myosin at the leader bleb neck that separates the leader bleb from the cell body (Fig. 1C \& Movie S2) (3-5). In concert with myosin, we wondered if the cortical actin flow in leader blebs requires the action of specific actin disassembly factors.

In addition to being frequently up-regulated in cancer, the ADF/cofilin family of actin severing factors are known to be essential for actin turnover in mesenchymal cells $(7,12)$. Therefore, we set out to determine if ADF and/or cofilin-1 are important for LBBM. Using melanoma A375 cells, a widely used cell line for the study of amoeboid migration, we depleted cells of ADF and cofilin-1 alone and together by RNAi (Fig. 1D \& S1A). By manually tracking the movement of cells over time, we found that cells depleted of cofilin-1 were significantly less motile (Fig. 1E-H). Additionally, the adhesive transmigration of cells through small pores was hindered after depleting cofilin-1 (Fig. S1B). Depleting cells of ADF led to a slight reduction in the number of highly motile cells (Fig. 1E-H). Strikingly, depleting cells of both ADF and cofilin-1 appeared to have an additive effect on reducing LBBM, whereas the adhesive transmigration of cells through small pores did not display this additive behavior and was not affected by ADF depletion (Fig. 1E-H \& S1B). These results suggest that these proteins play non-overlapping roles during LBBM (Fig. 1E-H). In agreement with this concept, transfection of EGFP-cofilin-1 into cells depleted of both proteins was insufficient to restore LBBM (Fig. 1E$\mathrm{H} \& \mathrm{~S} 1 \mathrm{~B})$.

\section{Together, ADF and cofilin-1 are required to retract blebs}

Subsequently, we wanted to know what is responsible for the decrease in LBBM upon depleting ADF and/or cofilin-1. Initially, we analyzed the area of the largest bleb (i.e., leader bleb), relative to the cell body, in cells depleted of cofilin-1. This analysis revealed that depleting cells 
of cofilin-1 reduced the area of the largest bleb to $\sim 30 \%$ of control (non-targeting; Fig. 2A-B \& Movies S3-5). The area of all blebs was similarly reduced (Fig. 2C). Strikingly, in cells depleted of both ADF and cofilin-1, $30 \%$ of cells displayed blebs with elongated necks (Fig. 2A, D \& Movie S6). Detailed analyses of these cells revealed that $\sim 60 \%$ display blebs that never retract into the cell body (Fig. $2 \mathrm{E}$ ). Moreover, we found this effect to not be specific to melanoma A375 cells, as depleting lung adenocarcinoma A549 cells of both ADF and cofilin-1 similarly resulted in the elongation of bleb necks (Fig. 2F-G). In melanoma A375 cells, depleting both $\mathrm{ADF}$ and cofilin-1 had an additive effect on reducing the rate of bleb retraction, which points to these proteins having non-overlapping functions specifically at bleb necks (Fig. $2 \mathrm{H})$. In support of this role, we determined the location of EGFP-cofilin-1 in confined cells. Our initial efforts were unsuccessful because of a high degree of soluble (i.e., unbound to Factin) protein, but in cells depleted of endogenous ADF and cofilin-1 we could detect an enrichment of EGFP-cofilin-1 at leader bleb necks (Fig. 2I; arrow).

\section{ADF and cofilin-1 rapidly disassemble cortical actin}

Thus far, our results suggest that ADF and cofilin-1 are important for the rapid turnover of cortical actin at bleb necks. In agreement with this concept, a large concentration of F-actin is found at the necks of blebs in cells depleted of ADF and cofilin-1 (Fig. 3A). In order to more directly test the role of ADF and cofilin-1 in regulating cortical actin, we turned to freshly trypsinized (spherical) cells. In spherical cells, actin is predominantly cortical and endogenous cofilin-1 is diffuse throughout the cytoplasm with some enrichment at the cell periphery (Fig. 3B). By combining the specificity of phalloidin for F-actin with flow cytometry, we then determined how the level of cortical actin is affected by ADF and/or cofilin-1 depletion. In cells depleted of cofilin-1, F-actin levels were increased by $\sim 10 \%$, whereas depletion of ADF did not lead to a significant change in the level of F-actin (Fig. 3C). Interestingly, depleting ADF with cofilin-1 had the largest effect, increasing F-actin levels by $\sim 30 \%$ (Fig. 3C). Serine 3 of cofilin-1 is phosphorylated by LIM Kinase (LIMK), inhibiting its severing activity (8). In cells depleted of endogenous ADF and cofilin-1, transfection of EGFP-cofilin-1 reduced the level of F-actin to levels similar to control (non-target; Fig. 3D). Similarly, F-actin was restored to near control levels by transfection of EGFP-cofilin-1 (S3A; constitutively active), whereas transfection of EGFP-cofilin-1 (S3E; constitutively inactive) led to an increased level of F-actin (Fig. 3D). Additionally, in comparison to EGFP alone, increasing levels of EGFP-cofilin-1 correlated with reductions in F-actin (Fig. 3E). Thus, cortical actin levels are regulated by ADF and cofilin-1 severing.

Many studies have shown that cofilin-1 severing can result in distinct outcomes. In lamellipodia, cofilin-1 severing promotes actin polymerization at new barbed ends, whereas in the lamella, actin is de-polymerized at new pointed ends (7). The prevalence of each outcome has been proposed to depend on the concentration of capping proteins (9). Therefore, we determined the relative level of barbed ends within the cortical actin network of freshly trypsinized (spherical) cells. The treatment of cells with cytochalasin B (which blocks G-actin 
from binding the barbed end) confirmed the specificity of the approach (Fig. S2A). In cells depleted of cofilin-1, we observed a more than $\sim 50 \%$ increase the level of cortical barbed ends, whereas depleting ADF marginally increased the level of cortical barbed ends (Fig. 3G). Depleting cells of both proteins appeared to have an additive effect, increasing the level of cortical barbed ends by $\sim 100 \%$ (Fig. 3G). However, in cells depleted of ADF and cofilin-1, transfection of EGFP-cofilin-1 was sufficient to restore cortical barbed ends to a level similar to control (Fig. 3H). Collectively, these results suggest that ADF and cofilin-1 regulate cortical actin levels by promoting the de-polymerization of actin at newly formed pointed ends.

\section{Rapid cortical actin flow requires ADF and cofilin-1 severing at leader bleb necks}

Lasting changes in the F/G-actin ratio, such as occurs during RNAi of ADF and/or cofilin-1, can have a range of effects on cellular physiology. Therefore, using a previously described SuperNova-cofilin-1 construct, we performed Chromophore Assisted Light Inactivation (CALI) for establishing the direct effects of cofilin-1 depletion $(13,14)$. In cells depleted of ADF and cofilin-1, SuperNova-cofilin-1 is predominantly observed at leader bleb necks (Fig. 4A). After 1 min of intense red light irradiation, we found that $~ 50 \%$ of SuperNova-cofilin- 1 is destroyed, as indicated by a reduction in red fluorescence (Fig. 4A). Using LifeAct-mEmerald for monitoring actin dynamics, we observed an accumulation of actin and the elongation of leader bleb necks within minutes of inactivating cofilin-1 (Fig. 4B \& Movie S7). Moreover, after inactivating SuperNova-cofilin-1, cortical actin flow rates in leader blebs were reduced by $\sim 50 \%$, whereas irradiation of SuperNova alone did not change actin flow rates (Fig. 4C-D \& S3A). Thus, as demonstrated by CALI, cofilin-1 directly regulates the cortical actin in leader blebs.

We then determined rates of actin turnover at leader bleb necks. For this, we used a version of LifeAct tagged with the photoactivable (green/red) fluorescent protein, mEos, for performing Fluorescence Loss After Photoactivation (FLAP) assays. Using a $405 \mathrm{~nm}$ laser, a pool of mEos-LifeAct was photoactivated at leader bleb necks. Subsequently, fluorescence decay at leader bleb necks was measured and used for determining rates of actin turnover (Fig 4E). To demonstrate proof of concept, curves were fit with a single phase decay, yielding an Rsquared value of 0.8348 (Fig. 4F). In contrast, a rapid decline in red fluorescence was not observed in paraformaldehyde treated cells (Fig. S3B). In control (non-target) cells, we found the actin at leader bleb necks to be rapidly turned over ( $\mathrm{t}_{1 / 2}$; Fig. $\left.4 \mathrm{G}\right)$. While the rate of actin turnover in cells depleted of cofilin-1 or ADF alone was similar to control (non-target), depleting both ADF and cofilin-1 led to a significant decrease in the actin turnover rate ( $\mathrm{t}_{1 / 2}$; Fig. $\left.4 \mathrm{G}\right)$. These data suggest that ADF and cofilin-1, together, are critical for the rapid turnover of cortical actin at leader bleb necks.

\section{Cofilin-1 supports both actin turnover and myosin contractility at leader bleb necks}

Because the cortical actin flow in leader blebs is driven by myosin, we wondered if ADF and/or cofilin-1 are important for cortical contractility. To address this possibility, we 
determined the compressibility of cells using a previously described gel sandwich approach (3). Briefly, freshly trypsinized (spherical) cells are placed between two polyacrylamide gels of known stiffness $(1 \mathrm{kPa})$. Subsequently, the ratio of the cell height $(h)$ to the diameter $(d)$ is used to determine compressibility (Fig. 5A). After depleting cofilin-1, we found cells to be significantly more compressible, whereas depleting ADF had no effect (Fig. 5A). Moreover, in cofilin-1 depleted cells, the depletion of ADF did not have any additional effect on cell compressibility (Fig. 5A). Therefore, cofilin-1 may be particularly important for contractility. In support of this idea, cofilin-1 has been shown to modulate contractility through a variety of mechanisms, which involve changes in both actin turnover and network architecture $(10,15,16)$. As determined by immunofluorescence imaging of phosphorylated Regulatory Light Chain (pRLC), we could confirm that signaling to myosin was not significantly affected by depleting ADF and/or cofilin-1 (Fig. 5B-C). In confined cells, depleting ADF and cofilin-1 leads to the elongation of leader bleb necks, which are decorated with myosin (RLC-EGFP; Fig. 5D). We then used CALI to determine if the accumulation of myosin at leader blebs necks is directly caused by the removal of these proteins. Indeed, within minutes of SuperNova-cofilin-1 inactivation, we observed myosin accumulating at leader bleb necks (RLC-EGFP; Fig. 5E \& Movie S8). Additionally, the flow of myosin towards leader bleb necks was significantly impeded after cofilin-1 inactivation, as determined by tracking individual myosin minifilaments (Fig. 5F). While ADF may be particularly important for actin turnover, these results point to cofilin-1 as having important roles in both actin turnover and myosin contractility at leader bleb necks.

\section{Discussion}

Here, we identify ADF and cofilin-1 as key mediators of the rapid (cortical) actin flow in leader blebs. This is significant as the rapid flow of cortical actin in leader blebs is essential for confined migration (3-5). We report that melanoma cells depleted of cofilin-1 poorly undergo LBBM, whereas removing ADF did not have a significant effect. Because ADF and cofilin-1 are thought to have redundant or overlapping roles, we were surprised to then find that depleting cofilin-1 together with ADF led to a near complete inhibition of LBBM. Therefore, we set out to determine the basis for this effect in cancer cells.

Initially, we used high spatial and temporal resolution imaging for close inspection of RNAi cells. Cells lacking cofilin-1 alone had much smaller blebs. This result is in line with previous work showing that cofilin-1 supports myosin contractility through optimizing actin filament lengths and de-branching $(10,15,16)$. Without both cofilin-1 and ADF, many cells had blebs with elongated necks that would not retract. A similar bleb defect has also been reported in HeLa cells (17). This led us to wonder if ADF and/or cofilin-1 may be important for actin turnover at bleb necks. In agreement with this concept, in cells lacking endogenous cofilin-1 and ADF, EGFP-cofilin-1 was enriched at bleb necks. 
In confined cells, we observed a dramatic accumulation of actin at bleb necks. In order to better understand how ADF and cofilin-1 regulate the overall level of cortical actin, we utilized spherical cells which predominantly have cortical actin. Using flow cytometry, we found that depleting cells of cofilin-1 could significantly increase the overall level of actin. Although ADF on its own had no effect, depleting cofilin-1 with ADF increased the overall level of actin even further, which might suggest that ADF augments the severing activity of cofilin-1. Similarly, the level of cortical barbed ends was significantly increased in the absence of cofilin-1, whereas removing ADF marginally increased the level of cortical barbed ends. Depleting both cofilin-1 and ADF appeared to have an additive effect, leading to the largest increase in the level of uncapped (polymerization competent) barbed ends. This result suggests that cofilin-1 severing, in collaboration with ADF, leads to the rapid disassembly of cortical actin and not polymerization at new barbed ends.

Because changes in the cellular F/G-actin ratio can trigger specific transcriptional programs, such as through the activation of the transcription factor, MRTF-A, we wanted to determine if the effects we observed on LBBM are a direct result of down regulating actin severing (18). For this, we utilized CALI for the rapid inactivation of SuperNova-cofilin-1. In cells depleted of ADF and cofilin-1, SuperNova-coflin-1 was sufficient to restore normal bleb morphology and dynamics. However, within minutes of cofilin-1 inactivation, actin began to accumulate at elongated bleb necks. By kymograph analysis, we also observed a significant decrease in the cortical actin flow rate. Using a photoactivatable LifeAct construct, we then directly measured the rate of actin turnover (i.e., fluorescence decay) at bleb necks. While depleting ADF or cofilin-1 alone did not have a significant effect on actin turnover rates, depleting both proteins led to a large increase in the rate of actin turnover at bleb necks. Thus, in cells lacking ADF and cofilin-1, defects in bleb morphology and dynamics correlate with a reduction in actin turnover at the neck.

Cofilin-1 has been previously implicated in regulating actomyosin contractility. Therefore, using spherical cells, we determined the overall effect of ADF and cofilin-1 on cortical contractility. As indicated by increased compressibility, cells were found to be less stiff after depleting cofilin-1. In contrast, removing ADF had little effect on cell stiffness. Moreover, depleting both ADF and cofilin-1 was similar to removing cofilin-1 alone. Therefore, cofilin-1 may be particularly important to support actomyosin contractility. In line with this notion, cofilin-1 has been shown to support myosin contractility through optimizing actin filament lengths and de-branching $(10,15,16)$. As indicated by the lack of any change in pRLC levels or localization, removing these proteins does not appear to effect signaling to myosin. The role of cofilin-1 in supporting myosin contractility is further supported by CALI. More specifically, we demonstrate that inactivating cofilin-1 decreases the rate at which myosin minifilaments flow towards the bleb neck.

Thus, our data are consistent with a model whereby ADF and cofilin-1 play key roles during LBBM. More specifically, we assert that ADF and cofilin-1, together, optimize actin 
disassembly and myosin contractility at bleb necks (Fig. 6, top). Whereas, in the absence of these proteins, incoming cortical actin fails to disassemble and accumulates with myosin at the elongated necks of persistent blebs (Fig. 6, below). Collectively, this study further points to rapid (cortical) actin flows as being essential for confined (leader bleb-based) migration. This is significant as many cancer cells have been shown to undergo $\operatorname{LBBM}(2,3)$.

This work also reveals an unanticipated role for ADF. In melanoma cells, ADF appears to augment the activity of cofilin-1 at bleb necks. Largely, ADF and cofilin-1 are thought to have redundant or overlapping roles. However, ADF has been shown to have significant monomer sequestering activity (19). We speculate that, in the absence of both proteins, dampened actin severing coupled with uncontrolled filament elongation contributes to the severe phenotypes we observe. Thus, the dissemination of melanoma tumors is likely to be blocked by the simultaneous inhibition of ADF and cofilin-1.

\section{Supplemental Information}

Supplemental information includes 3 figures and 8 movies and can be found with this article online.

\section{Methods}

Cell culture. A375-M2 (CRL-3223) and A549 (CCL-185) were obtained from the American Type Culture Collection (ATCC; Manassas, VA). Cells were cultured in high-glucose DMEM supplemented with 10\% FBS (cat no. 12106C; Sigma Aldrich, St. Louis, MO), GlutaMAX (Thermo Fisher, Carlsbad, CA), antibiotic-antimycotic (Thermo Fisher), and $20 \mathrm{mM} \mathrm{HEPES}$ at $\mathrm{pH} 7.4$ for up to 30 passages.

Confinement. This protocol has been described in detail elsewhere (11). Briefly, PDMS (cat no. 24236-10; Dow Corning 184 SYLGARD) was purchased from Krayden (Westminster, CO). 2 $\mathrm{mL}$ was cured overnight at $37^{\circ} \mathrm{C}$ in each well of a 6-well glass bottom plate (cat no. P06-1.5HN; Cellvis, Mountain View, CA). Using a biopsy punch (cat no. 504535; World Precision Instruments, Sarasota, FL), an $8 \mathrm{~mm}$ hole was cut and $3 \mathrm{~mL}$ of serum free media containing $1 \%$ BSA was added to each well and incubated overnight at $37{ }^{\circ} \mathrm{C}$. After removing the serum free media containing $1 \% \mathrm{BSA}, 300 \mu \mathrm{L}$ of complete media containing trypsinized cells $(250,000$ to 1 million) and $2 \mu \mathrm{L}$ of $3.11 \mu \mathrm{m}$ beads (cat no. PS05002; Bangs Laboratories, Fishers, IN) were then pipetted into the round opening. The vacuum created by briefly lifting one side of the hole with a $1 \mathrm{~mL}$ pipette tip was used to move cells and beads underneath the PDMS. Finally, $3 \mathrm{~mL}$ of complete media was added to each well and cells were recovered for $\sim 60$ min before imaging.

Plasmids. SuperNova-cofilin-1 was a gift from Dr. Kazuyo Sakai (Osaka University, Osaka, Japan). EGFP-cofilin-1 WT (no. 50859; a gift from Dr. James Bamburg), S3A (no. 50854; a gift from Dr. James Bamburg), S3E (no. 50855; a gift from Dr. James Bamburg), and mEos3.2- 
LifeAct (no. 54696; a gift from Michael Davidson) were obtained from Addgene (Watertown, MA). $1 \mu \mathrm{g}$ of plasmid was used to transfect 400,000 cells in each well of a 6 -well plate using Lipofectamine 2000 (5 $\mu \mathrm{L}$; Thermo Fisher) in OptiMEM (400 $\mu \mathrm{L}$; Thermo Fisher). After 20 min at room temperature, plasmid in Lipofectamine 2000/OptiMEM was then incubated with cells in complete media $(2 \mathrm{~mL})$ overnight.

Pharmacological treatments. Latrunculin-A (cat no. 3973) and cytochalasin B (cat no. 5474) were purchased from Tocris Bioscience (Bristol, UK). DMSO (Sigma Aldrich) was used to make $5 \mathrm{mM}$ and $2 \mathrm{mM}$ stock solutions of Latrunculin-A and cytochalasin B, respectively. To disassemble actin, cells resuspended in flow buffer were treated with $5 \mu \mathrm{M}$ Latrunculin A for 10 min at room temperature before flow cytometry. For barbed end assays, cytochalasin B was prediluted in complete media before it was incubated with cells for $1 \mathrm{hr}$ at $37{ }^{\circ} \mathrm{C}$.

LNAs. Non-targeting (cat no. 4390844), cofilin-1 (cat no. 4392420; s2936), and ADF (cat no. 4392422; s21737) Locked Nucleic Acids (LNAs) were purchased from Thermo Fisher. All LNA transfections were performed using RNAiMAX ( $5 \mu \mathrm{L}$; Thermo Fisher) and OptiMEM (400 $\mu \mathrm{L}$; Thermo Fisher). 100,000 cells were trypsinized and seeded in 6-well plates in complete media. After cells adhered ( $1 \mathrm{hr})$, LNAs in RNAiMAX/OptiMEM were added to cells in complete media $(2 \mathrm{~mL})$ at a final concentration of $50 \mathrm{nM}$. Cells were incubated with LNAs for two days.

Western blotting. Whole-cell lysates were prepared by scraping cells into ice cold RIPA buffer (50 mM HEPES pH 7.4,150 mM NaCl, 5 mM EDTA, 0.1\% SDS, 0.5\% deoxycholate, and 1\% Triton X-100) containing protease and phosphatase inhibitors (Roche, Switzerland). Before loading onto 4-12\% NuPAGE Bis-Tris gradient gels (Thermo Fisher), DNA was sheared by sonication and samples were boiled for $10 \mathrm{~min}$ in loading buffer. Following SDS-PAGE, proteins in gels were transferred to nitrocellulose membranes and subsequently immobilized by air drying overnight. After blocking in Tris-Buffered Saline containing 0.1\% Tween 20 (TBS-T) and $1 \%$ BSA, primary antibodies against ADF (cat no. MA5-25485; Thermo Fisher and cat no. D8818; Sigma Aldrich), and cofilin-1 (cat no. MA5-17275; Thermo Fisher) were incubated with membranes overnight at $4{ }^{\circ} \mathrm{C}$. Bands were then resolved with IRDye conjugated secondary antibodies on an Odyssey scanner from LI-COR Biosciences, Lincoln, NE. GAPDH (cat no. 97166; Cell Signaling Technology, Danvers, MA) was used to confirm equal loading.

Transmigration. Transmigration assays were performed using polycarbonate filters with 8 or 12 $\mu \mathrm{m}$ pores (Corning; Corning, NY). Prior to the assays, cells were serum starved for $24 \mathrm{hr}$ and polycarbonate filters were fibronectin $(10 \mu \mathrm{g} / \mathrm{mL}$; Millipore, Burlington, MA) coated for $1 \mathrm{hr}$ followed by air drying. 100,000 cells in serum free media were seeded in the top chamber while the bottom chamber contained media with $20 \%$ FBS to attract cells. After $24 \mathrm{hr}$, cells from the bottom of the filter were trypsinized and counted using an automated cell counter (TC20; Bio- 
Rad, Hercules, CA). Transmigration was then calculated as the ratio of cells on the bottom of the filter vs. the total.

Flow cytometry. Roughly $1 \times 10^{6}$ trypsinized cells in flow buffer (HBS with $1 \%$ BSA) were fixed using 4\% paraformaldehyde (cat no. 15710; Electron Microscopy Sciences, Hatfield, PA) for $20 \mathrm{~min}$ at room temperature. After washing, cell pellets were resuspended in flow buffer and incubated with $0.1 \%$ Triton X-100, Alexa Fluor 647-conjugated phalloidin (cat no. A22287; Thermo Fisher), and DAPI (Sigma Aldrich) for $30 \mathrm{~min}$ at room temperature. Data were acquired on a FACSCalibur (BD Biosciences, Franklin Lakes, NJ) flow cytometer. Flow cytometric analysis was performed using FlowJo (Ashland, OR) software.

Barbed end assay. The protocol for the barbed end assay was used with minor modifications (20,21). Prior to barbed end assays, cells were trypsinized and plated on poly-L-lysine coated 6well glass-bottom plates (Cellvis). To allow for a minimal level of cell attachment, cells were incubated for $10 \mathrm{~min}$ in a tissue culture incubator. Cells were then gently permeabilized for 1 min with saponin buffer $(138 \mathrm{mM} \mathrm{KCl}, 4 \mathrm{mM} \mathrm{MgCl}$, $3 \mathrm{mM}$ EGTA, $0.1 \%$ saponin, $1 \mathrm{mM}$ ATP, $3 \mu \mathrm{M}$ phalloidin, and 1\% BSA) followed by one wash with saponin-free buffer. Permeabilized cells were then incubated with Alexa Fluor 568-conjugated G-actin from rabbit muscle (cat no. A12374; Thermo Fisher) for 3 min in a tissue culture incubator and washed with saponin-free buffer. Treated cells were then fixed with $4 \%$ paraformaldehyde in Hepes-buffered saline (HBS), washed with HBS alone, and immediately imaged.

Immunofluorescence. After washing with Hepes-buffered saline (HBS), cells in 6-well glassbottom plates (Cellvis) were fixed with $4 \%$ paraformaldehyde (Electron Microscopy Sciences) in HBS for $20 \mathrm{~min}$ at room temperature. Blocking, permeabilization, antibody incubation, and washing were done in HBS with $1 \%$ BSA, $1 \%$ fish gelatin, $0.1 \%$ Triton X-100, and 5 mM EDTA. A 1:250 dilution of ADF (cat no. MA5-25485; Thermo Fisher), cofilin-1 (cat no. MA5-17275; Thermo Fisher), pRLC (cat no. PA5-17727 or MA5-15163; Thermo Fisher), or RLC (cat no. PA5-17624; Thermo Fisher) antibody was incubated with cells overnight at $4{ }^{\circ} \mathrm{C}$. After extensive washing, a 1:400 dilution of Alexa Fluor 488-conjugated anti-rabbit secondary antibody (cat no. A-21206; Thermo Fisher) was then incubated with cells for $2 \mathrm{hr}$ at room temperature. Cells were then incubated with a 1:250 dilution of Alexa Fluor 568-conjugated phalloidin (cat no. A12380; Thermo Fisher) and a 1:1000 dilution of DAPI (cat no. D1306; Thermo Fisher). Cells were again extensively washed and then imaged in HBS.

Cell stiffness assay. The protocol for the gel sandwich assay was used with minor modifications (3). 6-well glass bottom plates (Cellvis) and $18 \mathrm{~mm}$ coverslips were activated using 3aminopropyltrimethoxysilane (Sigma Aldrich) for $5 \mathrm{~min}$ and then for $30 \mathrm{~min}$ with $0.5 \%$ glutaraldehyde (Electron Microscopy Sciences) in PBS. $1 \mathrm{kPa}$ polyacrylamide gels were made using $2 \mu \mathrm{L}$ of blue fluorescent beads ( $200 \mathrm{~nm}$; Thermo Fisher), $18.8 \mu \mathrm{L}$ of $40 \%$ acrylamide 
solution (cat no. 161-0140; Bio-Rad), and $12.5 \mu \mathrm{L}$ of bis-acrylamide (cat no. 161-0142; Bio$\mathrm{Rad}$ ) in $250 \mu \mathrm{L}$ of PBS. Finally, $2.5 \mu \mathrm{L}$ of Ammonium Persulfate (APS; $10 \%$ in water) and 0.5 $\mu \mathrm{L}$ of Tetramethylethylenediamine (TMED) was added before spreading $9 \mu \mathrm{L}$ drops onto treated glass under coverslips. After polymerizing for $40 \mathrm{~min}$, the coverslip was lifted in PBS, extensively rinsed and incubated overnight in PBS. Before each experiment, the gel attached to the coverslip was placed on a $14 \mathrm{~mm}$ diameter, $2 \mathrm{~cm}$ high PDMS column for applying a slight pressure to the coverslip with its own weight. Then, both gels were incubated for $30 \mathrm{~min}$ in media before plates were seeded. After the bottom gels in plates was placed on the microscope stage, the PDMS column with the top gel was placed on top of the cells seeded on the bottom gels, confining cells between the two gels. After $1 \mathrm{hr}$ of adaptation, the height of cells was determined with beads by measuring the distance between gels, whereas the cell diameter was measured using a far-red plasma membrane dye (cat no. C10046; Thermo Fisher). Stiffness was defined as the height $(h)$ divided by the diameter $(d)$.

Microscopy. Live high-resolution imaging was performed using a General Electric (Boston, MA) DeltaVision Elite imaging system mounted on an Olympus (Japan) IX71 stand with a computerized stage, environment chamber (heat, $\mathrm{CO}_{2}$, and humidifier), ultrafast solid-state illumination with excitation/emission filter sets for DAPI, CFP, GFP, YFP, and Cy5, critical illumination, Olympus PlanApo N 60X/1.42 NA DIC (oil) objective, Photometrics (Tucson, AZ) CoolSNAP HQ2 camera, proprietary constrained iterative deconvolution, and vibration isolation table.

Chromophore Assisted Light Inactivation (CALI). Live confined cells co-transfected with SuperNova-cofilin-1 and either mEmerald-LifeAct or EGFP-RLC were imaged every 12 sec for $5 \mathrm{~min}$ before CALI. Cells were then subjected to $1 \mathrm{~min}$ of high intensity red light irradiation (DeltaVision Elite). Images were subsequently acquired every $12 \mathrm{sec}$ for $1 \mathrm{hr}$ post-irradiation.

Actomyosin flow rates. Actin and myosin minifilament flow rates were calculated from images taken every $12 \mathrm{sec}$ of mEmerald-LifeAct and EGFP-RLC, respectively. Kymographs of each were generated in Fiji (https://imagej.net/Fiji) and spanned the length of leader blebs.

Fluorescence Loss After Photobleaching (FLAP). Cells transiently transfected with the photoactivatable construct, mEos3.2-LifeAct, were imaged using a Zeiss (Germany) laser scanning confocal microscope (LSM880) with fast Airy Scan. Regions at bleb necks were photo-converted using the $405 \mathrm{~nm}$ laser at $20 \%$ power (3 iterations). Regions were sampled every $15 \mathrm{~ms}$ for 10 and 150 cycles before and after photo-conversion, respectively. Fluorescence measurements were acquired using ZEN software (Zeiss) and decay was calculated as $\mathrm{F} / \mathrm{F}_{0}$. Non-linear one-phase decay curves were fit to data using GraphPad Prism. 
Cell migration. To perform cell speed and plot of origin analyses, we used an Excel (Microsoft, Redmond, WA) plugin, DiPer, developed by Gorelik and colleagues and the Fiji plugin, MTrackJ, developed by Erik Meijering for manual tracking (22,23). For minimizing positional error, cells were tracked every other frame. Brightfield imaging was used to confirm that beads were not obstructing the path of a cell. Cells that traveled a distance equivalent to at least one cell length over the course of the $5 \mathrm{hr}$ time-lapse were classified as highly motile.

Bleb morphology and dynamics. For leader bleb and total bleb areas, freshly confined cells were traced from high-resolution images with the free-hand circle tool in Fiji (https://imagej.net/Fiji). From every other frame, the percentage of cell area for leader blebs and percentage of cell area for total blebs were calculated in Excel (Microsoft). Frame-by-frame measurements were then used to generate an average for each cell. Bleb retraction rates were determined by dividing the bleb length by the amount of time taken to completely retract the bleb into the cell body. For each cell, retraction rates were calculated from 2-3 blebs.

Statistics. All box plots are Tukey in which "+" and line denote the mean and median, respectively. Sample sizes were determined empirically and based on saturation. As noted in each figure legend, statistical significance was determined by either a two-tailed Student's t-test or multiple-comparison test post-hoc. Normality was determined by a D'Agostino \& Pearson test in GraphPad Prism. * $-\mathrm{p} \leq 0.05, * *-\mathrm{p} \leq 0.01$, *** $-\mathrm{p} \leq 0.001$, and **** $-\mathrm{p} \leq 0.0001$

Illustration. The model for ADF and cofilin-1 function was drawn in BioRender (Toronto, ON).

Data availability. The data that support the findings of this study are available from the corresponding author, J.S.L., upon reasonable request.

\section{Acknowledgements}

We thank members of the Logue Lab for insightful discussions and especially, Dr. Sandrine B. Lavenus, for help with cell stiffness measurements. We would also like to thank the administrative staff within the Department of Regenerative and Cancer Cell Biology at the Albany Medical College. This work was supported by a Young Investigator Award from the Melanoma Research Alliance (MRA; award no. 688232).

\section{Author Contributions}

J.S.L. conceived and designed the study. M.F.U. performed all laboratory research. M.F.U and J.S.L. wrote the manuscript.

\section{Competing Financial Interests}

The authors declare no competing financial interests. 


\section{References}

1. Pollard, T. D., and Borisy, G. G. (2003) Cellular motility driven by assembly and disassembly of actin filaments. Cell 112, 453-465

2. Logue, J. S., Cartagena-Rivera, A. X., Baird, M. A., Davidson, M. W., Chadwick, R. S., and Waterman, C. M. (2015) Erk regulation of actin capping and bundling by Eps8 promotes cortex tension and leader bleb-based migration. Elife 4

3. Liu, Y. J., Le Berre, M., Lautenschlaeger, F., Maiuri, P., Callan-Jones, A., Heuze, M., Takaki, T., Voituriez, R., and Piel, M. (2015) Confinement and low adhesion induce fast amoeboid migration of slow mesenchymal cells. Cell 160, 659-672

4. Ruprecht, V., Wieser, S., Callan-Jones, A., Smutny, M., Morita, H., Sako, K., Barone, V., Ritsch-Marte, M., Sixt, M., Voituriez, R., and Heisenberg, C. P. (2015) Cortical contractility triggers a stochastic switch to fast amoeboid cell motility. Cell 160, 673-685

5. Bergert, M., Erzberger, A., Desai, R. A., Aspalter, I. M., Oates, A. C., Charras, G., Salbreux, G., and Paluch, E. K. (2015) Force transmission during adhesion-independent migration. Nat Cell Biol 17, 524-529

6. Charras, G. T., Coughlin, M., Mitchison, T. J., and Mahadevan, L. (2008) Life and times of a cellular bleb. Biophys J 94, 1836-1853

7. Bravo-Cordero, J. J., Magalhaes, M. A., Eddy, R. J., Hodgson, L., and Condeelis, J. (2013) Functions of cofilin in cell locomotion and invasion. Nature reviews Molecular cell biology 14, 405

8. Yang, N., Higuchi, O., Ohashi, K., Nagata, K., Wada, A., Kangawa, K., Nishida, E., and Mizuno, K. (1998) Cofilin phosphorylation by LIM-kinase 1 and its role in Rac-mediated actin reorganization. Nature 393, 809-812

9. Wioland, H., Guichard, B., Senju, Y., Myram, S., Lappalainen, P., Jégou, A., and RometLemonne, G. (2017) ADF/Cofilin accelerates actin dynamics by severing filaments and promoting their depolymerization at both ends. Current Biology 27, 1956-1967. e1957

10. Chan, C., Beltzner, C. C., and Pollard, T. D. (2009) Cofilin dissociates Arp2/3 complex and branches from actin filaments. Current Biology 19, 537-545

11. Logue, J., Chadwick, R., and Waterman, C. (2018) A simple method for precisely controlling the confinement of cells in culture.

12. Bracalente, C., Rinflerch, A. R., Ibanez, I. L., Garcia, F. M., Volonteri, V., Galimberti, G. N., Klamt, F., and Duran, H. (2018) Cofilin-1 levels and intracellular localization are associated with melanoma prognosis in a cohort of patients. Oncotarget 9, 24097-24108

13. Vitriol, E. A., Wise, A. L., Berginski, M. E., Bamburg, J. R., and Zheng, J. Q. (2013) Instantaneous inactivation of cofilin reveals its function of F-actin disassembly in lamellipodia. Mol Biol Cell 24, 2238-2247

14. Takemoto, K., Matsuda, T., Sakai, N., Fu, D., Noda, M., Uchiyama, S., Kotera, I., Arai, Y., Horiuchi, M., Fukui, K., Ayabe, T., Inagaki, F., Suzuki, H., and Nagai, T. (2013) SuperNova, a monomeric photosensitizing fluorescent protein for chromophore-assisted light inactivation. Sci Rep 3, 2629

15. Chugh, P., Clark, A. G., Smith, M. B., Cassani, D. A., Dierkes, K., Ragab, A., Roux, P. P., Charras, G., Salbreux, G., and Paluch, E. K. (2017) Actin cortex architecture regulates cell surface tension. Nature cell biology 19, 689 
16. Ennomani, H., Letort, G., Guérin, C., Martiel, J.-L., Cao, W., Nédélec, F., Enrique, M., Théry, M., and Blanchoin, L. (2016) Architecture and connectivity govern actin network contractility. Current Biology 26, 616-626

17. Shaw, A. E., DeLuca, J. G., and Bamburg, J. R. (2012) ADF/cofilin regulates actomyosin assembly through competitive inhibition of myosin II binding to F-actin. Developmental cell 22, 530-543

18. Olson, E. N., and Nordheim, A. (2010) Linking actin dynamics and gene transcription to drive cellular motile functions. Nat Rev Mol Cell Biol 11, 353-365

19. Chen, H., Bernstein, B. W., Sneider, J. M., Boyle, J. A., Minamide, L. S., and Bamburg, J. R. (2004) In vitro activity differences between proteins of the ADF/cofilin family define two distinct subgroups. Biochemistry-Us 43, 7127-7142

20. Vitriol, E. A., Uetrecht, A. C., Shen, F., Jacobson, K., and Bear, J. E. (2007) Enhanced EGFP-chromophore-assisted laser inactivation using deficient cells rescued with functional EGFP-fusion proteins. Proceedings of the National Academy of Sciences 104, 6702-6707

21. Symons, M. H., and Mitchison, T. J. (1991) Control of actin polymerization in live and permeabilized fibroblasts. The Journal of cell biology 114, 503-513

22. Gorelik, R., and Gautreau, A. (2014) Quantitative and unbiased analysis of directional persistence in cell migration. Nat Protoc 9, 1931-1943

23. Meijering, E., Dzyubachyk, O., and Smal, I. (2012) Methods for cell and particle tracking. Methods Enzymol 504, 183-200 


\section{Figures}
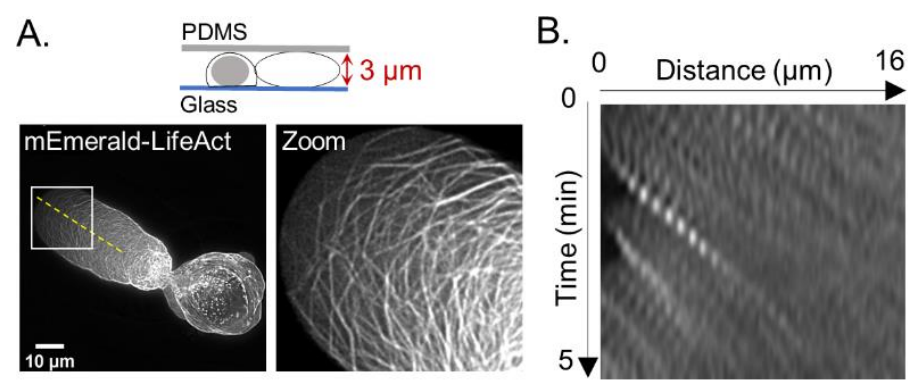

C.

EGFP-RLC
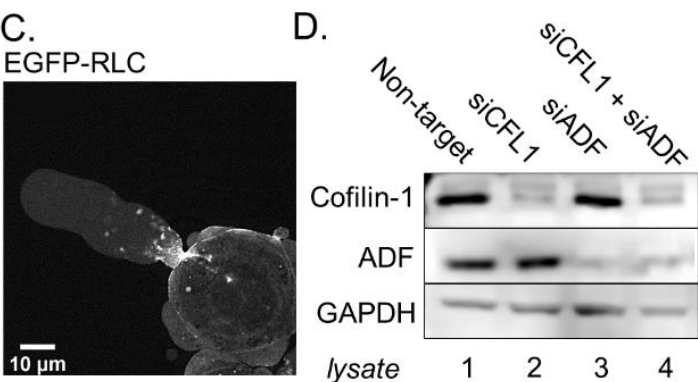

E.
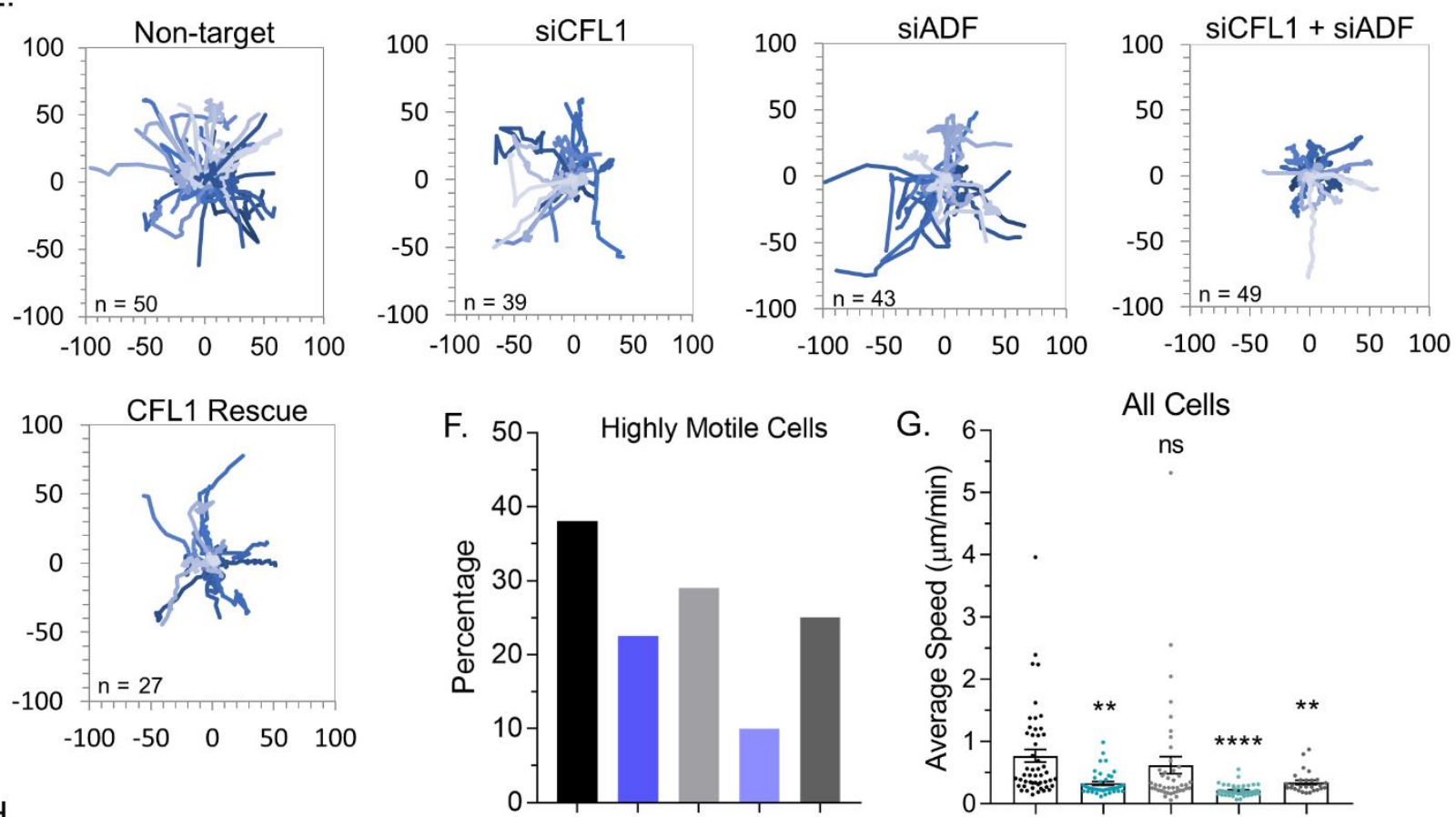

G.

All Cells

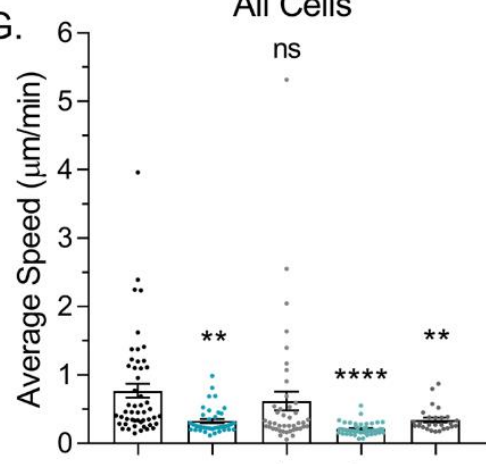

$\mathrm{H}$.

Highly Motile Cells
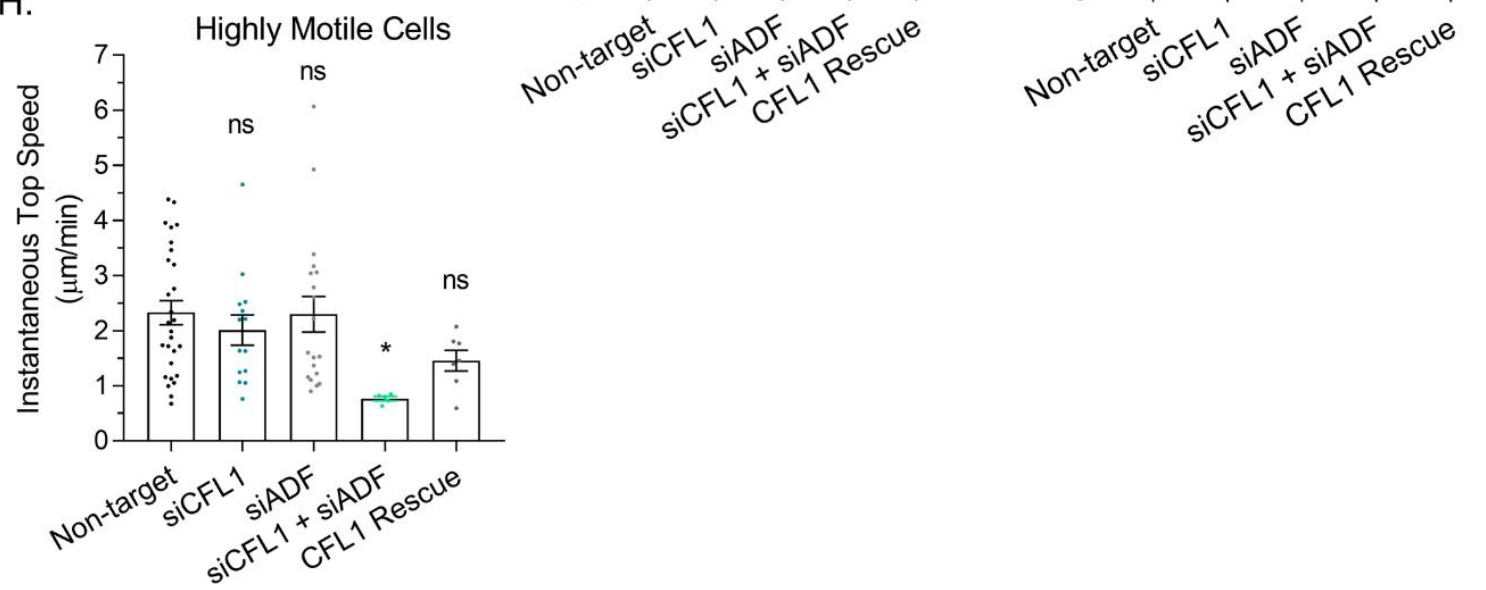

Figure 1. ADF and cofilin-1 are required for leader bleb-based migration. A. Ventral Zsection of a melanoma A375-M2 cell, which has been confined down to $3 \mu \mathrm{m}$, with mEmeraldLifeAct. B. Kymograph from (A; dashed line), showing cortical actin flow. C. Ventral Zsection of a melanoma A375-M2 cells, which has been confined down to $3 \mu \mathrm{m}$, with EGFP- 
RLC. D. Western blot confirming CFL1, ADF, and ADF + CFL1 RNAi in melanoma A375-M2 cells. E. Individual cell migration tracks (plot of origin) for non-targeting, CFL1, ADF, and CFL1 + ADF RNAi cells, as well as, CFL1 + ADF RNAi cells rescued by transfection with EGFP-cofilin-1 plasmid. In each, cells were tracked over a period of $5 \mathrm{hr}$. Relative y $(\mu \mathrm{m})$ and relative $\mathrm{x}(\mu \mathrm{m})$ are shown in each. F. Percentage of highly motile cells from $(\mathrm{E})$. Cells that traveled a distance equivalent to at least one cell length over the course of the $5 \mathrm{hr}$ time-lapse were classified as highly motile. G. Average speed $(\mu \mathrm{m} / \mathrm{min})$ from cells in (E; mean $+/-\mathrm{SEM})$. Statistical significance was determined by one-way ANOVA and a Dunnett's post-hoc test. $\mathbf{H}$. Instantaneous top speed $(\mu \mathrm{m} / \mathrm{min})$ for highly motile cells in $(\mathrm{E}$; mean $+/-\mathrm{SEM})$. Statistical significance was determined by one-way ANOVA and a Dunnett's post-hoc test. All data are representative of at least three independent experiments. * $-\mathrm{p} \leq 0.05, * *-\mathrm{p} \leq 0.01, * * *-\mathrm{p} \leq$ 0.001 , and $* * * *-\mathrm{p} \leq 0.0001$ 


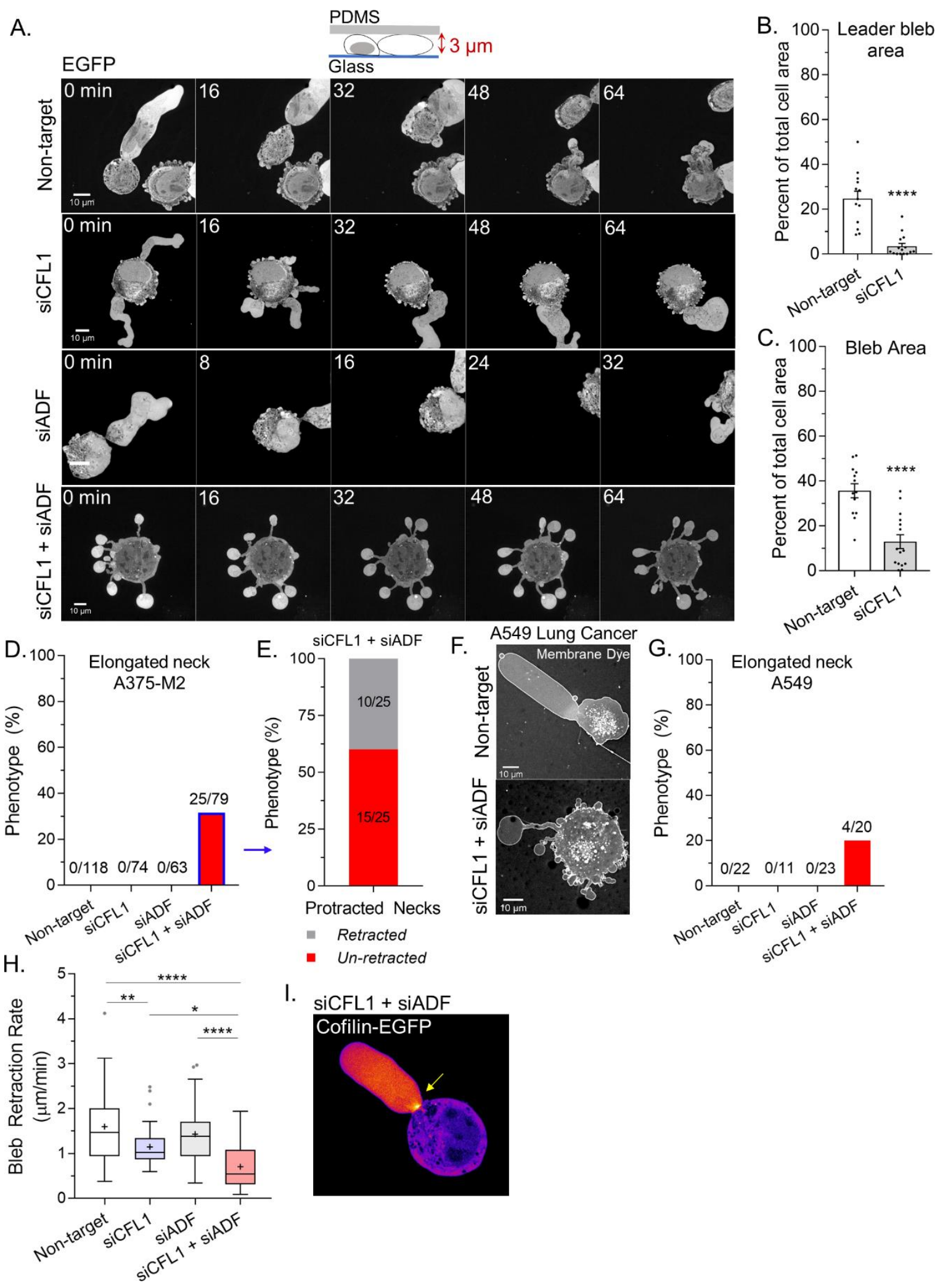

Figure 2. Together, ADF and cofilin-1 are required to retract blebs. A. Montage of nontargeting, CFL1, ADF, and CFL1 + ADF RNAi with EGFP alone (volume marker) in melanoma 
A375-M2 cells. B-C. Quantitation of area for leader (A) and all blebs (B) after non-targeting and CFL1 RNAi. Statistical significance was determined using an unpaired Student's t-test. D. Percent of non-targeting, CFL1, ADF, and CFL1 + ADF RNAi cells with elongated bleb necks. E. Percent of ADF + CFL1 RNAi cells from (D) with elongated bleb necks that retract vs. unretracted. F-G. Lung adenocarcinoma A549 cells after non-targeting and CFL1 + ADF RNAi stained with a far-red fluorescent membrane dye (F). Percent of non-targeting, CFL1, ADF, and CFL1 + ADF RNAi cells with elongated bleb necks $(\mathrm{G})$. H. Bleb retraction rates for nontargeting (45 blebs; 26 cells), CFL1 (40 blebs; 20 cells), ADF (48 blebs; 30 cells), and CFL1 + ADF RNAi (38 blebs; 23 cells). Statistical significance was determined by one-way ANOVA and a Dunnet's post-hoc test. I. EGFP-cofilin-1 localization in an A375-M2 cell confined down to $3 \mu \mathrm{m}$. Arrow points to an enrichment of cofilin-1 at the leader bleb neck. All data are representative of at least three independent experiments. * $-\mathrm{p} \leq 0.05, * *-\mathrm{p} \leq 0.01, * * *-\mathrm{p} \leq$ 0.001 , and $* * * *-p \leq 0.0001$ 


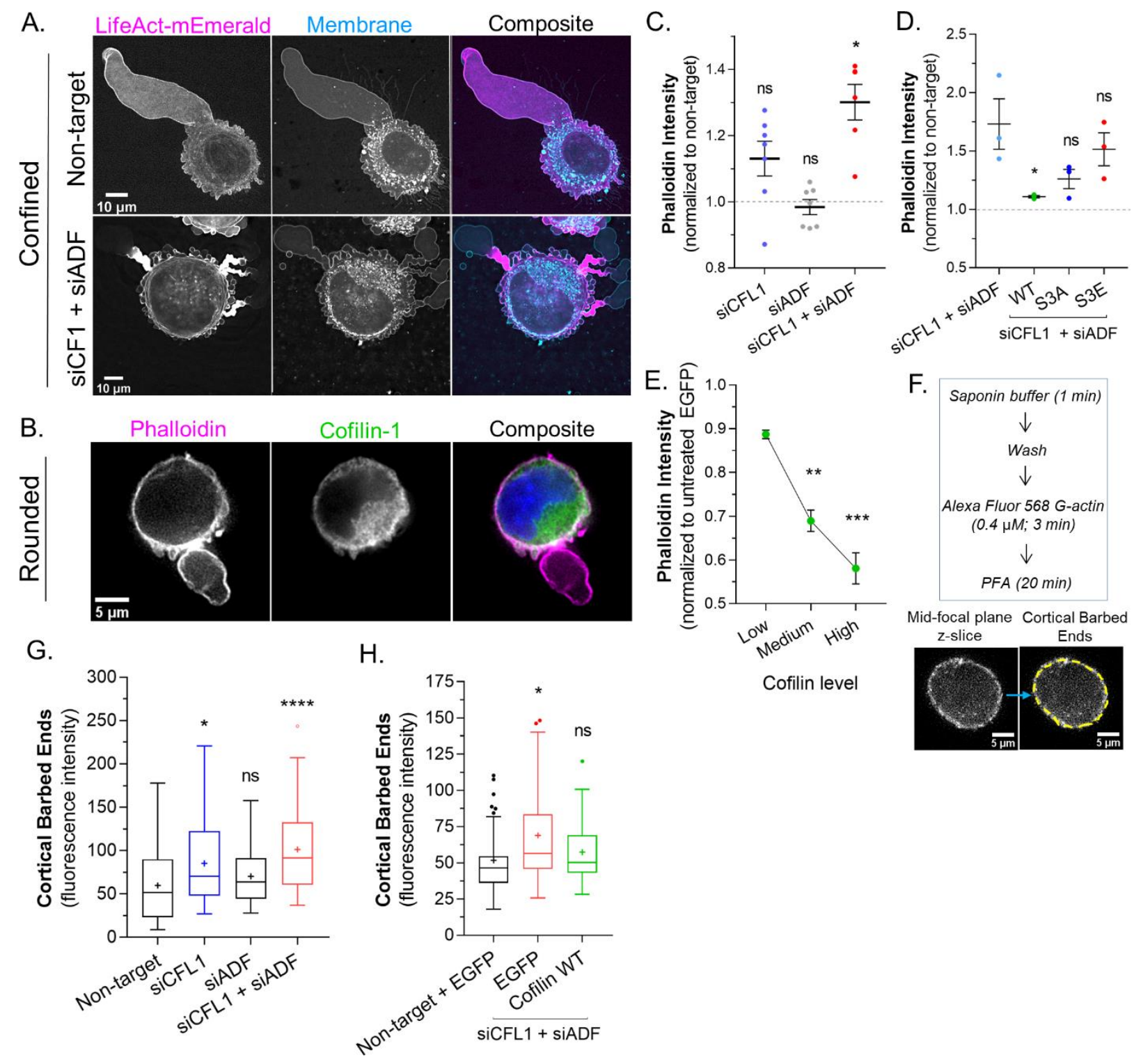

Figure 3. ADF and cofilin-1 rapidly disassemble cortical actin. A. mEmerald-LifeAct and far-red fluorescent membrane dye in cells after non-targeting and CFL1 + ADF RNAi. B. Cells freshly plated on poly-L-lysine coated cover-glass stained for endogenous cofilin-1 and F-actin (phalloidin). C. F-actin levels (normalized to non-target; mean +/- SEM) after CFL1, ADF, and CFL1 + ADF RNAi in trypsinized (spherical) cells, as determined by flow cytometry. Statistical significance was determined by one-way ANOVA and a Dunnet's post-hoc test. D. F-actin levels (normalized to non-target; mean +/- SEM) after CFL1 + ADF RNAi, as well as, after CFL1 + ADF RNAi with EGFP-cofilin-1 WT, S3A, or S3E, as determined by flow cytometry. Statistical significance was determined by one-way ANOVA and a Dunnet's post-hoc test. E. Factin level (normalized to EGFP alone; mean +/- SEM) as a function of increasing EGFP-cofilin1 in cells depleted of endogenous cofilin-1 and ADF by RNAi, as determined by flow cytometry. Statistical significance was determined by one-way ANOVA and a Dunnet's post-hoc test. F. 
Top, barbed end assay workflow. Bottom, representative image of a freshly plated (spherical) cell subjected to the barbed end assay. G. As shown in (F; bottom), the level of cortical barbed ends was measured in cells after non-targeting (71 cells), CFL1 (53 cells), ADF (47 cells), and CFL1 + ADF RNAi (83 cells). Statistical significance was determined by one-way ANOVA and a Dunnet's post-hoc test. H. As shown in (F; bottom), the level of cortical barbed ends was measured in cells with non-targeting and EGFP (42 cells), as well as, after CFL1 + ADF RNAi with EGFP (32 cells) or EGFP-cofilin-1 (27 cells). Statistical significance was determined by one-way ANOVA and a Dunnet's post-hoc test. All data are representative of at least three independent experiments. $*-\mathrm{p} \leq 0.05, * *-\mathrm{p} \leq 0.01, * * *-\mathrm{p} \leq 0.001$, and $* * * *-\mathrm{p} \leq 0.0001$ 

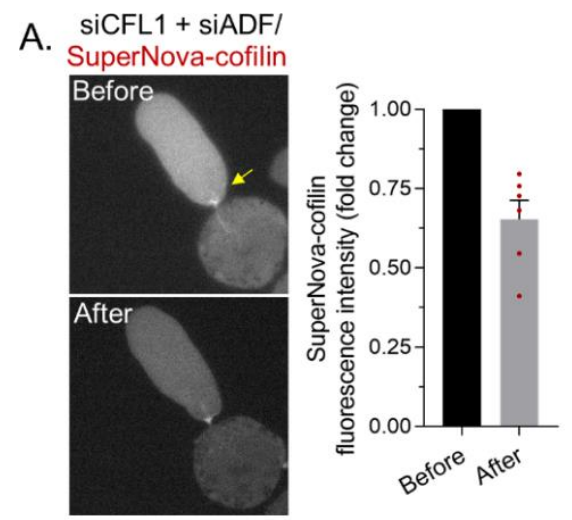

c.

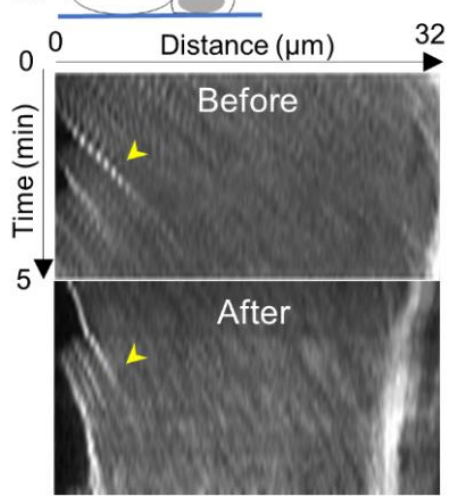

F.

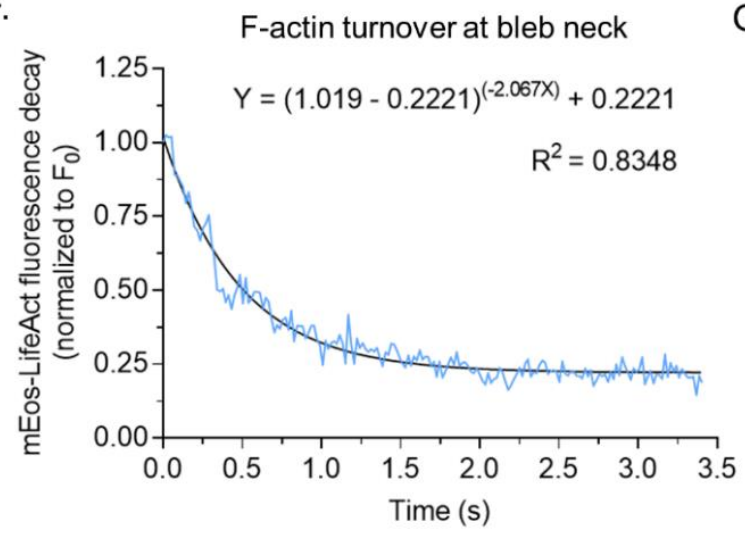

B. Time $(\mathrm{s}) \longrightarrow$ CALI-mediated inactivation (1 min)

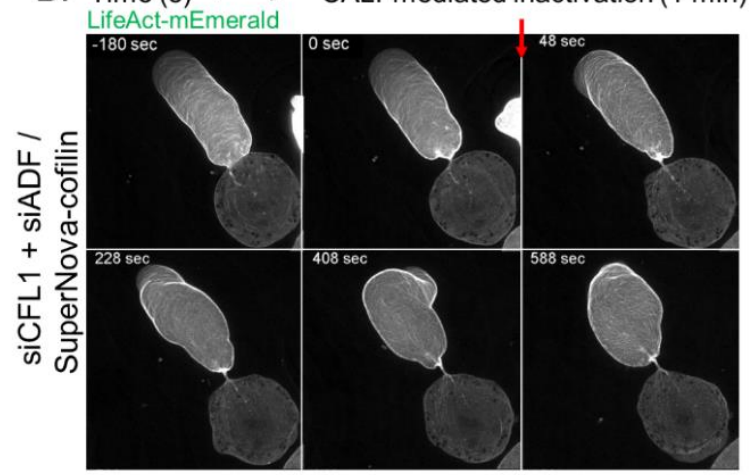

D.

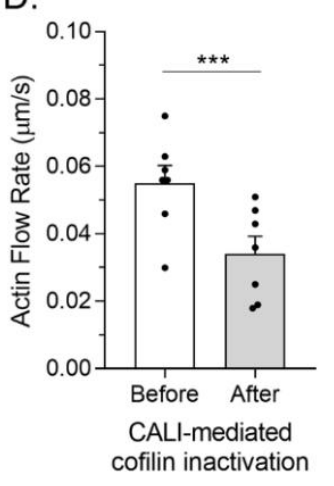

E.

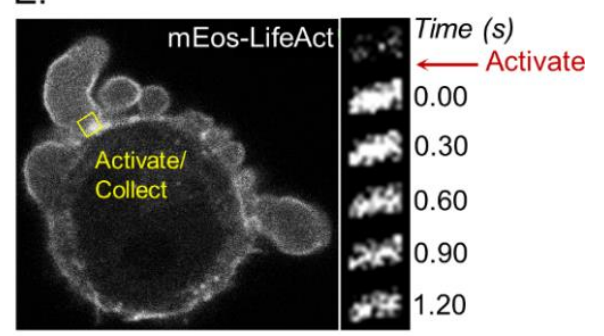

F-actin turnover at

G. 1.0 bleb neck

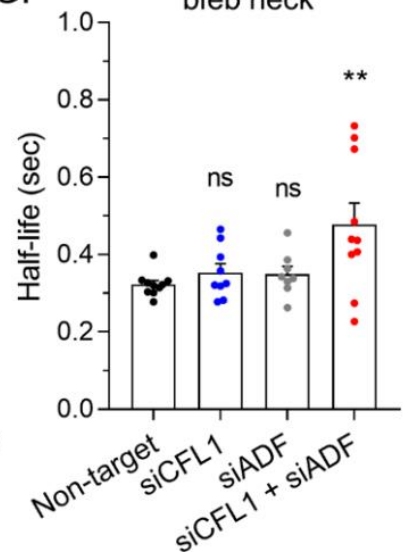

Figure 4. Rapid cortical actin flow requires ADF and cofilin-1 severing at leader bleb necks. A. Left, SuperNova-cofilin-1 localization in cells depleted of endogenous cofilin-1 and ADF by RNAi before and after 1 min of red light irradiation. Right, quantitative analysis of CALI, as determined by the fold change in SuperNova emission. B. Montage of mEmeraldLifeAct before and after cofilin-1 inactivation in a cell depleted of endogenous cofilin-1 and ADF by RNAi. C. Kymographs of cortical actin (mEmerald-LifeAct) flow from the leader bleb tip before and after cofilin-1 inactivation. D. Quantitative evaluation of cortical actin flow rates before and after cofilin-1 inactivation. Statistical significance was determined by a paired Student's t-test. E. Left, representative image of a freshly plated (spherical) cells with mEos3.2- 
LifeAct. Right, montage of mEos3.2-LifeAct within the shown ROI before and after photoactivation. F. Average decay curve for mEos3.2-LifeAct at bleb necks (normalized to the initial fluorescence level; $\mathrm{F} / \mathrm{F}_{0}$ ). The curve was fit using a non-linear single phase decay function. G. $\mathrm{T}_{1 / 2}$ for mEos3.2-LifeAct after photoactivation at bleb necks for non-targeting, CFL1, ADF, and CFL1 + ADF RNAi. Statistical significance was determined by one-way ANOVA and a Dunnet's post-hoc test. All data are representative of at least three independent experiments. $*-\mathrm{p} \leq 0.05, * *-\mathrm{p} \leq 0.01, * * *-\mathrm{p} \leq 0.001$, and $* * * *-\mathrm{p} \leq 0.0001$ 

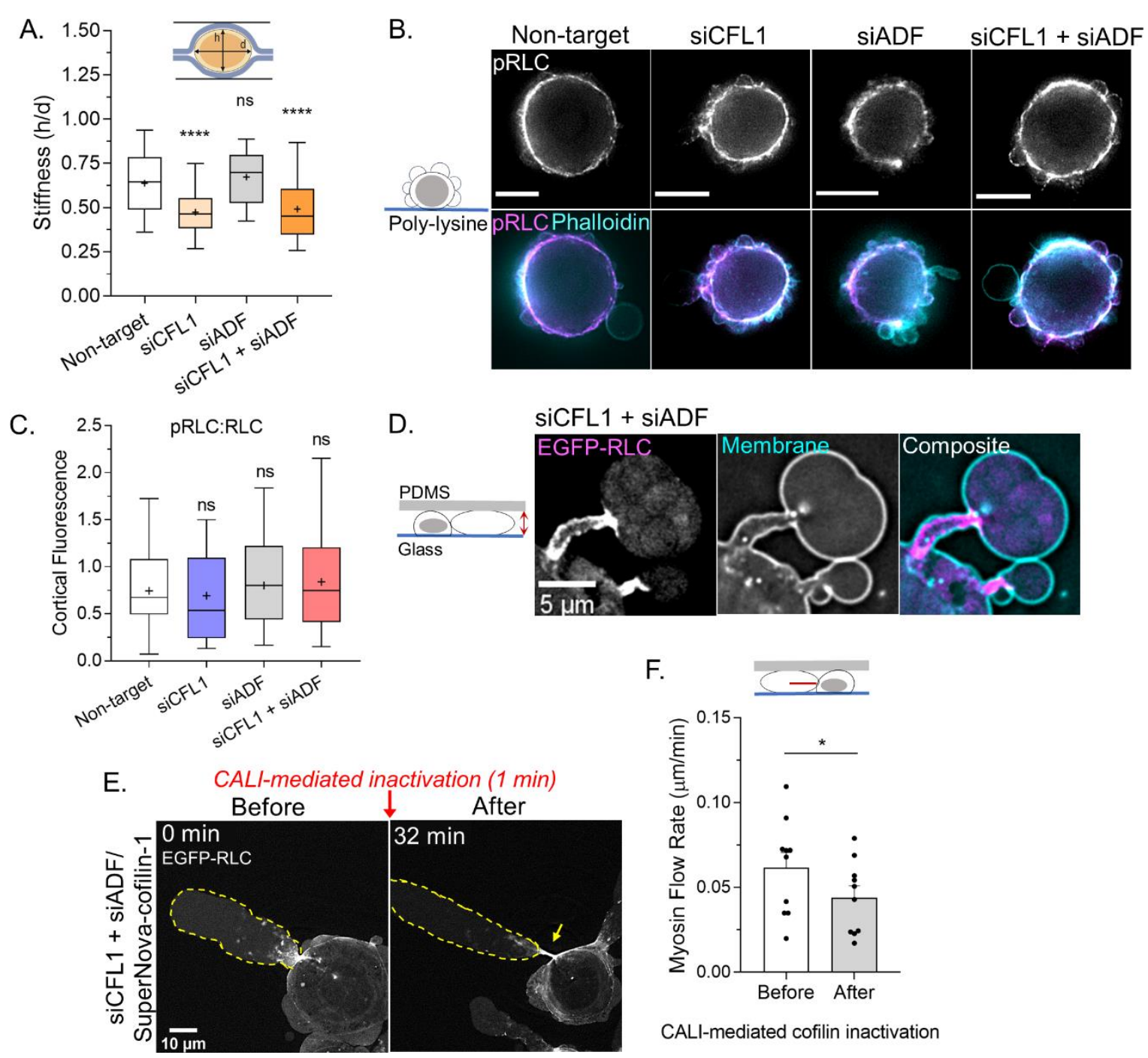

Figure 5. Cofilin-1 supports both actin turnover and myosin contractility at leader bleb

necks. A. A previously described gel sandwich assay was used to measure the stiffness $(h / d)$ of spherical cells after non-targeting (91 cells), CFL1 (30 cells), ADF (25 cells), and CFL1 + ADF RNAi (42 cells). Statistical significance was determined by one-way ANOVA and a Dunnet's post-hoc test. B. Immunofluorescence imaging of endogenous pRLC (S18), total RLC, and Factin (phalloidin) in freshly plated (spherical) cells after non-targeting, CFL1, and CFL1 + ADF RNAi. C. Ratio of cortical pRLC (S18) to total RLC fluorescence intensity after non-targeting (114 cells), CFL1 (107 cells), ADF (124 cells), and CFL1 + ADF RNAi (91 cells). Statistical significance was determined by one-way ANOVA and a Dunnet's post-hoc test. D. Localization of EGFP-RLC in a cell confined down to $3 \mu \mathrm{m}$ after CFL1 + ADF RNAi. E. EGFP-RLC dynamics in a cell depleted of cofilin-1 and ADF before and after CALI of SuperNova-cofilin-1. Arrow points to myosin accumulating at an elongating leader bleb neck after cofilin-1 inactivation. F. Myosin minifilament flow rate before and after cofilin-1 inactivation. Statistical significance was determined using a paired Student's t-test. All data are representative of at least 
bioRxiv preprint doi: https://doi.org/10.1101/2021.01.11.426289; this version posted February 24, 2021. The copyright holder for this preprint (which was not certified by peer review) is the author/funder, who has granted bioRxiv a license to display the preprint in perpetuity. It is made available under aCC-BY-NC-ND 4.0 International license.

three independent experiments. * $-\mathrm{p} \leq 0.05, * *-\mathrm{p} \leq 0.01, * * *-\mathrm{p} \leq 0.001$, and $* * * *-\mathrm{p} \leq$ 0.0001 


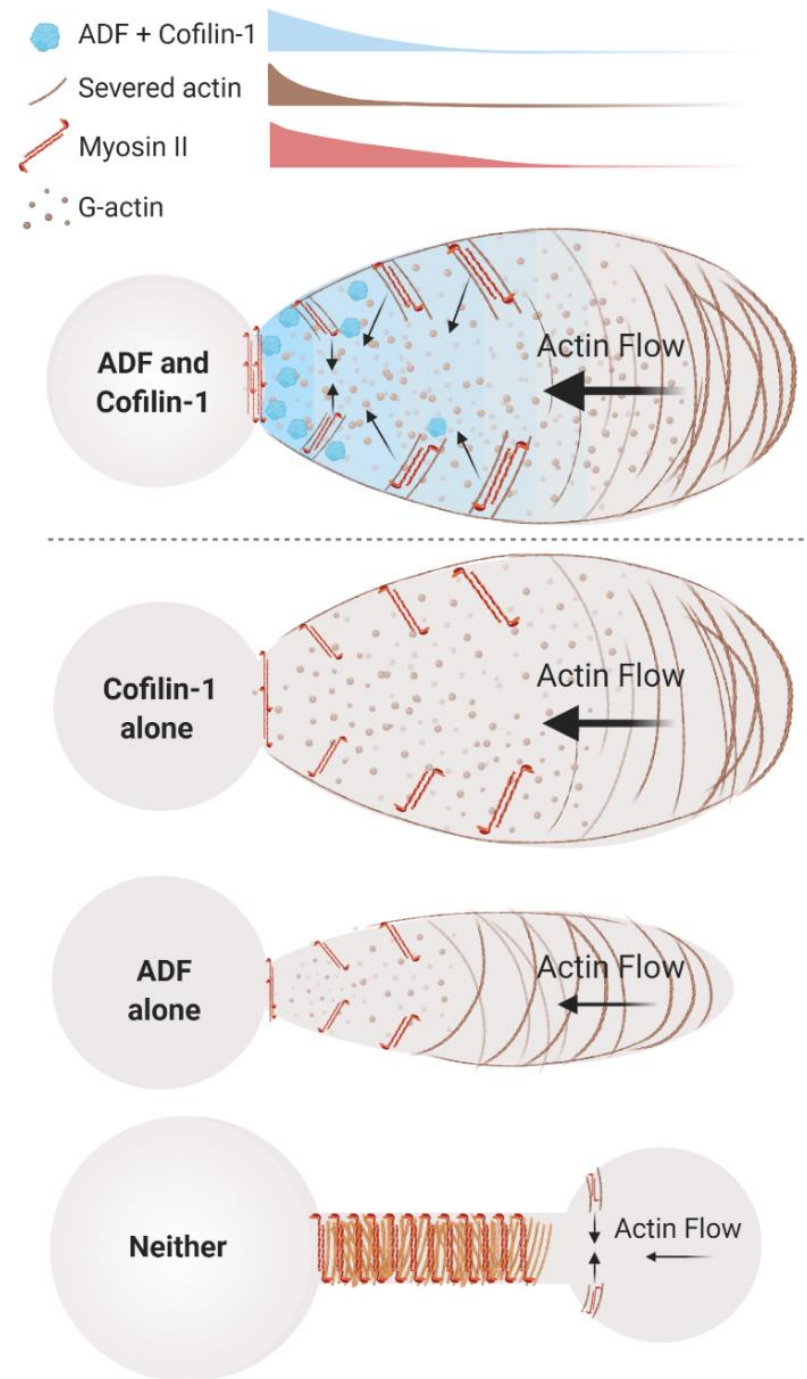

Figure 6. Model of ADF and cofilin-1 function within leader blebs. Top, in the presence of both ADF and cofilin-1, cells display large blebs with rapid cortical actin flow. Below, in the absence of cofilin-1 or ADF, cells form smaller blebs with slower cortical actin flow. Bottom, without both $\mathrm{ADF}$ and cofilin-1, blebs display several defects, including a failure to retract and an accumulation of actomyosin at elongated necks. 


\section{Supplemental Figures}
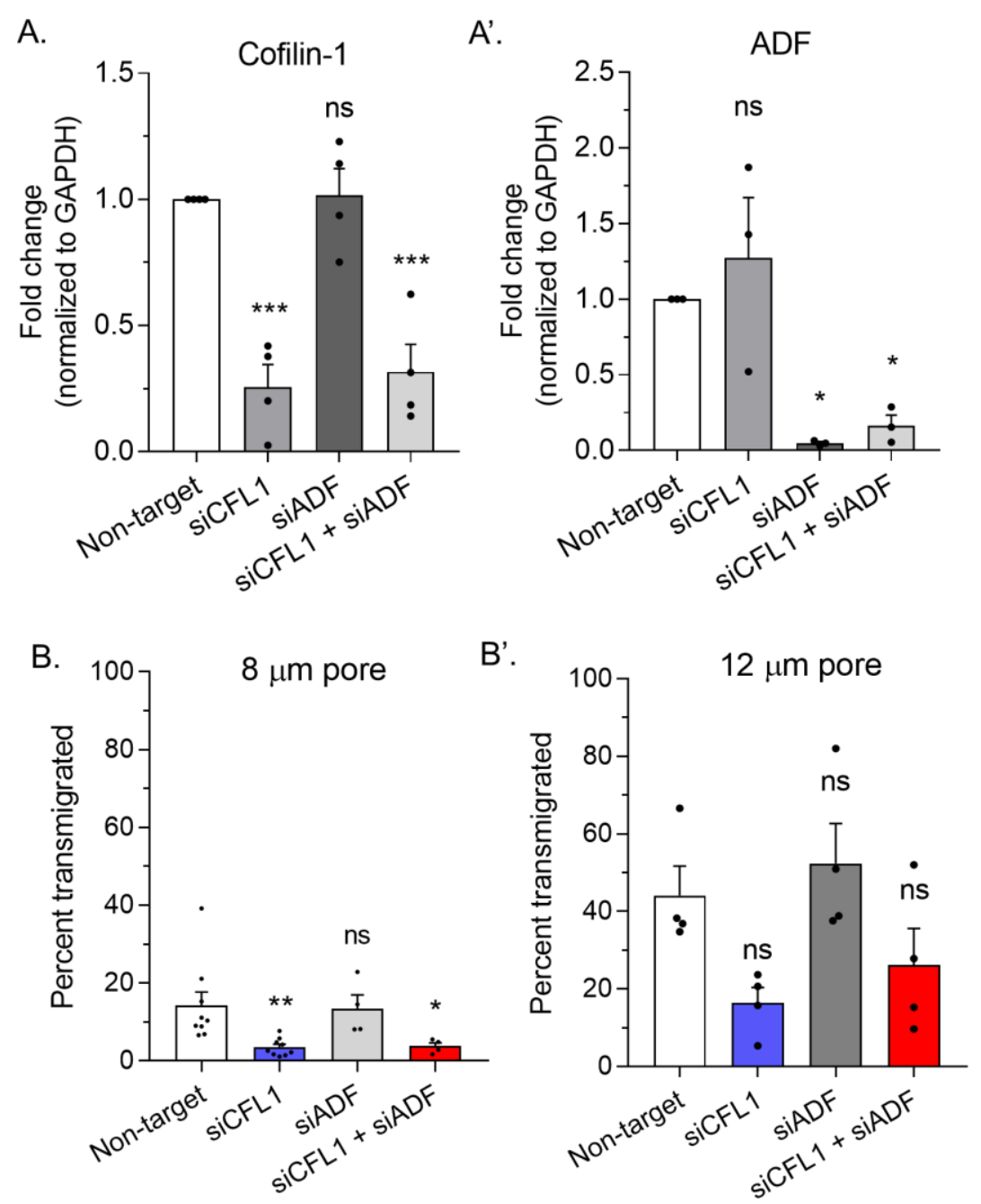

Supplemental figure 1. A. Quantitative evaluation of cofilin-1 (left) and ADF (right) RNAi as measured by Western blotting of total cell lysates. B. Transmigration of melanoma A375-M2 cells through 8 (left) or $12 \mu \mathrm{m}$ (right) pores after non-targeting, CFL1, ADF, and CFL1 + ADF RNAi. Statistical significance was determined by one-way ANOVA and a Dunnet's post-hoc test. All data are representative of at least three independent experiments. * - $\mathrm{p} \leq 0.05,{ }^{* *}-\mathrm{p} \leq$ $0.01, * * *-p \leq 0.001$, and $* * * *-p \leq 0.0001$ 

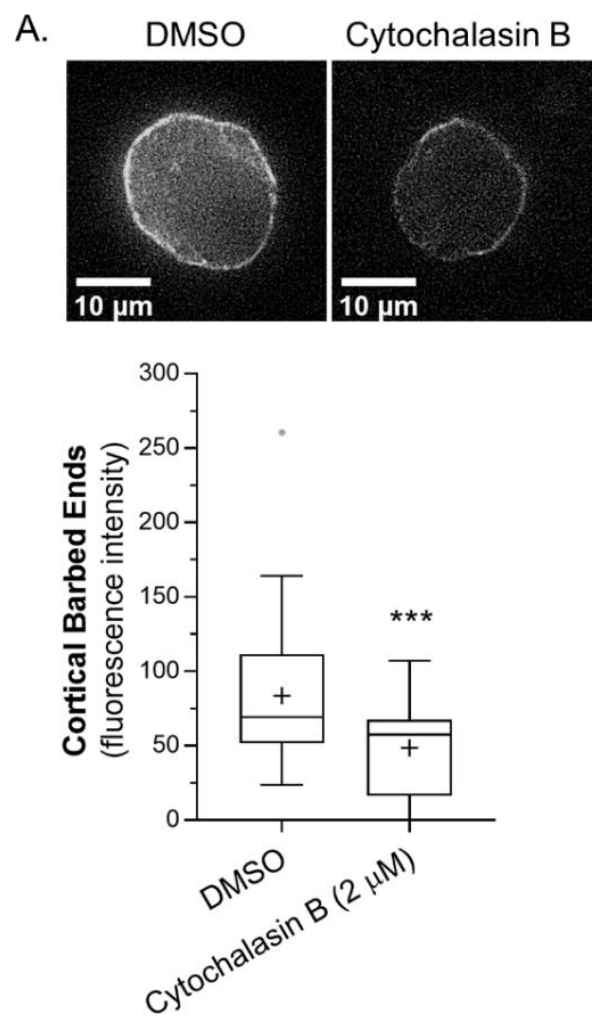

Supplemental figure 2. A. Cortical barbed end levels in melanoma A375-M2 cells after vehicle (DMSO; 53 cells) or cytochalasin B $(2 \mu \mathrm{M} ; 24$ cells) treatment. Statistical significance was determined using an unpaired Student's t-test. All data are representative of at least three independent experiments. * - $\mathrm{p} \leq 0.05, * *-\mathrm{p} \leq 0.01, * * *-\mathrm{p} \leq 0.001$, and $* * * *-\mathrm{p} \leq 0.0001$ 
A.

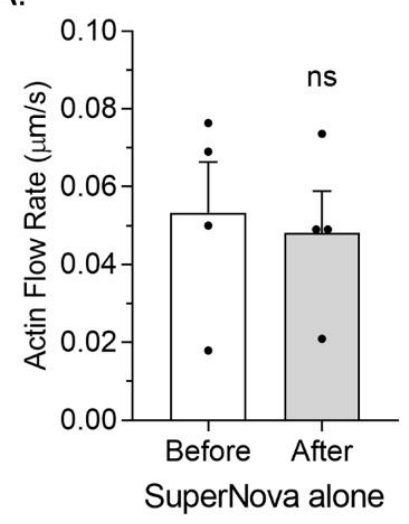

B.
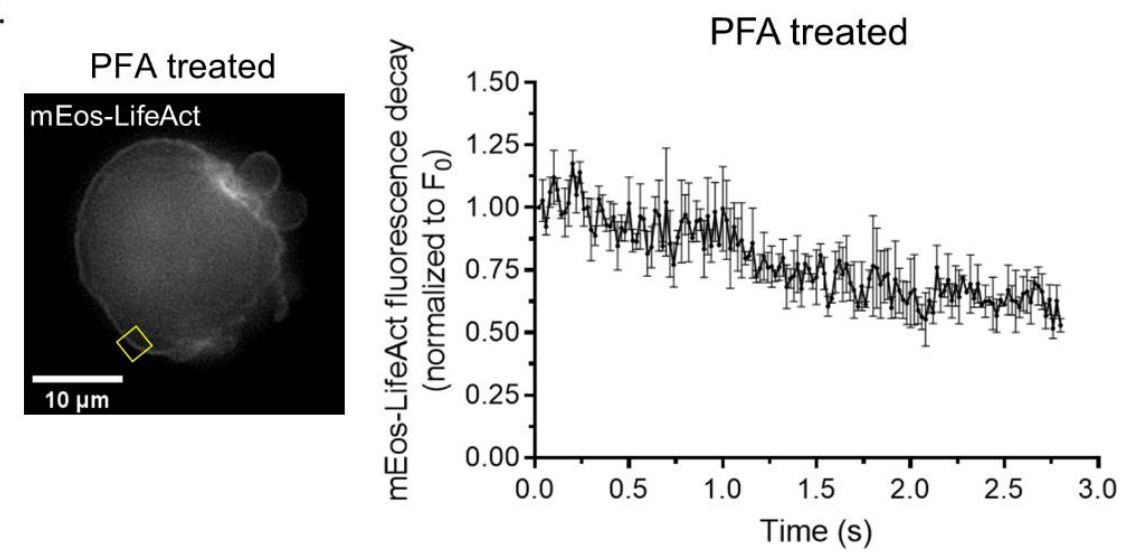

Supplemental figure 3. A. Cortical actin (mEmerald-LifeAct) flow rates before and after the inactivation (i.e., red light irradiation) of SuperNova alone. B. Left, sample ROI from a Paraformaldehyde (PFA) treated melanoma A375-M2 cell with mEos3.2-LifeAct. Right, average mEos3.2-LifeAct decay (3 cells; mean +/- SEM) after photoactivation. All data are representative of at least three independent experiments. 


\section{Supplemental Movies}

Supplemental movie 1. Time-lapse imaging of melanoma A375-M2 cells confined down to 3 $\mu \mathrm{m}$ with far red plasma membrane dye.

Supplemental movie 2. Time-lapse imaging of a melanoma A375-M2 cell confined down to 3 $\mu \mathrm{m}$ with mScarlet-LifeAct and EGFP-RLC.

Supplemental movie 3. Time-lapse imaging of a melanoma A375-M2 cell confined down to 3 $\mu \mathrm{m}$ with the volume marker, mScarlet, after control (non-targeting) RNAi.

Supplemental movie 4. Time-lapse imaging of a melanoma A375-M2 cell confined down to 3 $\mu \mathrm{m}$ with the volume marker, EGFP, after RNAi of ADF alone.

Supplemental movie 5. Time-lapse imaging of a melanoma A375-M2 cell confined down to 3 $\mu \mathrm{m}$ with the volume marker, mScarlet, after RNAi of CFL1 alone.

Supplemental movie 6. Time-lapse imaging of a melanoma A375-M2 cell confined down to 3 $\mu \mathrm{m}$ with the volume marker, EGFP, after RNAi of CFL1 + ADF.

Supplemental movie 7. Time-lapse imaging of a melanoma A375-M2 cell confined down to 3 $\mu \mathrm{m}$ with mEmerald-LifeAct after CALI of SuperNova-cofilin-1. The cell was depleted of endogenous ADF and cofilin-1 by RNAi.

Supplemental movie 8. Time-lapse imaging of a melanoma A375-M2 cell confined down to 3 $\mu \mathrm{m}$ with EGFP-RLC after CALI of SuperNova-cofilin-1. The cell was depleted of endogenous ADF and cofilin-1 by RNAi. 